\title{
Mehr Markt denn Nachhaltigkeit
}

\author{
Auch im Bereich der europäischen Forschungsförderung wird nachhaltige Ent- \\ wicklung als ein vorrangiges, durch Forschung zu unterstützendes Ziel formu- \\ liert. Doch wie konkretisieren sich die Forschungsaufgaben für eine nachhaltige \\ Entwicklung wirklich? Welches Verständnis von nachhaltiger Entwicklung liegt \\ den Forschungsprogrammen zugrunde? Und welche Perspektiven für Nachhal- \\ tigkeitsforschung sind in den nächsten Jahren zu erwarten?
}

$\mathrm{N}$ Von Nina Sartori eben den nationalen Programmen gewinnt die Forschungsförderung der Europäischen Union an Bedeutung. Die Umsetzung einer nachhaltigen Entwicklung ist ein zentrales Anliegen der EU. Im Vierjahres-Rhythmus legt die Kommission Forschungsrahmenprogramme auf, die aufgrund sich verändernder politischer Prioritätensetzungen unterschiedliche Schwerpunkte haben. Bis 2010 soll Europa der wettbewerbsfähigste wissensbasierte Wirtschaftsraum der Welt werden. Dies wurde auf dem Gipfel des Europarates in Lissabon im Jahr 2000 als zentrales Ziel festgehalten. Das derzeit laufende 6. Rahmenprogramm, wurde als entscheidendes Werkzeug konzipiert, um die wissenschaftlich-technologischen Grundlagen der Industrie und die Entwicklung ihrer Wettbewerbsfähigkeit zu stärken und um Forschungsmaßnahmen für andere Politikbereiche zu unterstiitzen. Die inhaltliche Ausrichtung der europäisch geförderten Forschung ist stark technologie- und marktorientiert. ,Wissen“ und ,Wissensgesellschaft" werden vor allem im Sinne eines Wettbewerbsvorsprungs interpretiert.

\section{Auftrag: mehr Wettbewerbs- fähigkeit}

Für das 6. Rahmenprogramm wurde von Ex-Forschungskommissar Philippe Busquin die Verwirklichung des ,Europäischen Forschungsraumes' (EFR) als neues Ziel formuliert. Durch eine verstärkte und effizientere Bündelung und Strukturierung der europäischen Forschungsanstrengungen und -kapazitäten soll das Ziel von Lissabon erreicht werden. Konkret bedeutet dies, dass neue Formen von Großprojekten geschaffen wurden, welche die gesamte europäische Spitzenforschung eines spezifischen Bereiches unter dem Dach eines einzigen groß angelegten Forschungsprojektes oder -netzes vereinigen sollen. Gleichzeitig wurden die Sozialund Geisteswissenschaften mit einem eigenen the-
Veränderungen und Ökosysteme“. Hier heißt es weiter: „Im Vertrag wird die nachhaltige Entwicklung als zentrales Ziel der Europäischen Gemeinschaft ausgewiesen. Dies ... findet in der Strategie der Union für die nachhaltige Entwicklung ... seinen Niederschlag. In diesem Kontext sind globale Veränderungen, Energieversorgungssicherheit, Nachhaltigkeit im Verkehr, nachhaltige Nutzung der natürlichen Ressourcen Europas und die Wechselwirkungen mit menschlichen Tätigkeiten zentrale Anliegen dieses vorrangigen Themenbereichs".

Dieser ,,vorrangige Themenbereich“ hat drei Schwerpunkte: i) Nachhaltige Energiesysteme, ii) Nachhaltiger Land- und Seeverkehr und iii) Globale Veränderungen und Ökosysteme. Hier stehen Umwelt- und Naturschutz im Mittelpunkt. Zwar ist dies ein Beitrag zu einer nachhaltigen Entwicklung, ökonomische und soziale Ziele werden jedoch nur am Rande und als Zusatzformulierung erwähnt. Ziel des Schwerpunktes „Nachhaltige Energiesysteme" beispielsweise ist, verbesserte Technologien für alternative Energiequellen zur Marktreife zu führen und damit einen Umwelt- und Wettbewerbsbeitrag zu leisten. Die Themen sind stark auf Technologieentwicklung, -verbesserung und Wettbewerbsfähigkeit ausgerichtet. Nur am Rande werden „,sozioökonomische Konzepte für umweltfreundlichen Strom“ als Forschungsthema eingebaut.

Je weiter sich die Themen in ihren Arbeitsprogrammen und Ausschreibungen konkretisieren, desto weniger bleibt vom oben zitierten Grundsatz, auch „Wechselwirkungen mit menschlichen Tätigkeiten“ zu berücksichtigen, übrig. In den jeweiligen Arbeitsprogrammen, wo die Themen für die laufenden Ausschreibungen beschrieben werden, werden multidisziplinäre Ansätze nur geringfügig eingefordert. Auch in der thematischen Priorität für die Sozial- und Geisteswissenschaften „Bürger und Staat in der Wissensgesellschaft" konzentriert sich die Auseinandersetzung mit nachhaltiger Entwicklung auf die Frage nach geeigneten Governance-Modellen.

\section{Entwicklungen und Perspektiven}

Sprechen Sie genau Ihre Zielgruppe an. Erscheint 5 x jührlich.

Fordern Sie noch heute die Mediadaten an!
Kontakt:

Dagmar Huber

Waltherstraße 29,80337 München

Fax 089/544 18449

E-Mail huber@oekom.de
Der Konzipierung der thematischen Priorität „Nachhaltige Entwicklung, globale Veränderungen und Ökosysteme" liegen auch der Aktionsplan für Umwelttechnologie (ETAP: Environmental Action Plan) und das 6. Umweltaktionsprogramm der EG zugrunde. Letzteres schlägt einen sehr marktorientierten Ansatz vor, in dem es einfordert, dass Umwelterwägungen integraler Bestandteil von unter- 
nehmerischen Denken werden sollten und entscheidend beim Kaufverhalten von Konsumenten. Ziel des ETAP ist es, allein Technologien zu fördern, ,um einerseits ökologischen Herausforderungen zu begegnen und andererseits Wettbewerbsfähigkeit und Wachstum zu fördern“. Darunter fallen Technologien und Verfahren, die zur direkten Verringerung der Umweltverschmutzung führen. Auf die Notwendigkeit, auch soziale und ökonomische Fragestellungen zu berücksichtigen, wird zwar verwiesen, doch bietet das sechste Rahmenprogramm dafür kaum konkrete Anknüpfungspunkte.

Eine Neuerung innerhalb des sechste Rahmenprogramms war sicherlich die Konzipierung einer thematischen Priorität für sozioökonomische und in sehr begrenztem Maße auch geisteswissenschaftliche Fragestellungen. Dies hat starke positive Resonanz in der Forschungslandschaft gefunden, dieser Bereich weist auch die höchste Überzeichnung auf. Aber es hat auch zu einer ,Technologisierung“ der anderen thematischen Prioritäten geführt. Durch die Bündelung der soziökonomischen Forschung ist das Prinzip der Interdisziplinarität ein Stückweit aufgegeben worden und hat die starke Fokussierung auf Technologieentwicklung des sechsten Rahmenprogramms ermöglicht.

Das vorangegangene fünfte Rahmenprogramm vertrat demgegenüber einen problemlösungsorientierten Ansatz. Europäische Forschung sollte zwar auch technologische und wirtschaftliche Grundlagen verbessern, aber vor allem helfen, gesellschaftliche Probleme in Europa zu lösen. Sozioökonomische Aspekte waren integraler Bestandteil aller thematischen Prioritäten, Budgetanteile für solche transdisziplinäre Forschung waren klar ausgewiesen. Das ermöglichte insgesamt eine ganzheitlichere Herangehensweise an Forschungsgebiete. Im Bereich der Umweltforschung und nachhaltigen Entwicklung war insbesondere beim Thema Energie ein Forschungsfeld sozialwissenschaftlichen Fragestellungen gewidmet: Sozio-ökonomische Aspekte von Energie im Rahmen einer Nachhaltigen Entwicklung (Wirkungen auf Gesellschaft, Wirtschaft und Beschäftigung). Ein Ansatz, der in dieser Reichweite im 6. Rahmenprogramm völlig fehlt. Im Bereich Informationstechnologien lag ein klarer Schwerpunkt auf gesellschaftlichem Nutzen, Mitbestimmungsmöglichkeiten und sozialer Inklusion.

\section{Das 7. Rahmenprogramm}

Für das kommende siebte Forschungsrahmenprogramm von 2006 bis 2010 verfolgt die Kommission sechs große Zielsetzungen:

- Thematische Prioritäten werden weiterhin eine tragende Rolle spielen, um durch Kooperation „europäische Pole der Exzellenz“ zu schaffen.

- Europäische Technologie-Plattformen sollen als strukturierende Maßnahmen eingesetzt werden um Innovationen und Schlüsseltechnologien zu fördern.

- Die Grundlagenforschung wird innerhalb eines europäischen Forschungsrates möglich sein, um Wettbewerb zwischen Teams auf europäischer Ebene zu erreichen. Exzellenz wird als einziges Förderungskriterium angewandt werden.

- Europa soll attraktiver für die besten WissenschaftlerInnen werden.
- Das siebte Rahmenprogramm soll weiterhin die Schaffung des Europäischen Forschungsraumes vorantreiben und die Forschungsinfrastrukturen ausbauen.

- Nationale Forschungsprogramme sollen stärker koordiniert werden.

In dieser Prioritätenliste steht Nachhaltige Entwicklung nicht auf der Agenda, die Technologie- und Marktorientierung der Forschungsförderung wird weiter verstärkt. Dennoch bieten sich verschiedene Anknüpfungspunkte: Die Beibehaltung der thematischen Prioritäten sichert zumindest einen Forschungsbereich für Umwelttechnologien. Auch die zukünftige Förderung der Grundlagenforschung bietet für eine Forschung für die Nachhaltigkeit neue Möglichkeiten. Vor allem könnte aber die stärkere Vernetzung nationaler Programme eine weitere Ausbreitung des Forschungsansatzes der transdisziplinären Nachhaltigkeits-Forschung ermöglichen. Deutschland nimmt in diesem Bereich eine Vorreiterrolle ein und könnte inhaltliche und methodische Anregungen für die anderen Mitgliedsstaaten und die Kommission liefern.

\section{Die Autorin}

Nina Sartori ist Mitarbeiterin der Nationalen Kontaktstelle "Wissensgesellschaft" im EU-Büro des BMBF. Kontakt: BMBF-EU-Büro, PT-DLR, Königswinterer Str. 522-524, 53227 Bonn. Tel. 0228-447 645, E-Mail: nina.sartori@dlr.de

\section{GALA - Ókologlsche Perspekttwen In Natur-, Gelstes- und WIrtschaftswiksenschaften}

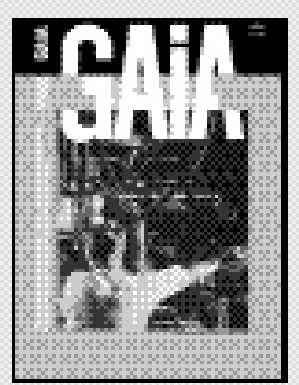

\section{Kommunales Nach haltigkeitsmanagement}

Dis Agends 21 sctreibt den Kommunen eine Sclvassololls bei der Umeatsung des Letbilks einer nacthaltigen Entwicklung zu. Oft korrmt deser Prosess in Kormunalpoltik und -wsrusltung jedoch nur schloppord voran. Ulich Gehrlein (fior deutsche Kornmuren' sowie lenas Fridker und Witsutorinnien (far de Schweiz) zeigen neue W'oge auf und prssentieren effektive und effizisnte Modelle des barnmunalen Machihaltigkeitsmanagoments. Lesen Sio hierzu auch einen kritischen Kommentar won Lars Holtkamp.

Außerdem in der aktuellen ALEgabe von GALA 4/04:

Jachhattigbeitsforschung - jenseits von Desiplinisrung und ayything goes (Benjamin Nolting ot al.?

Stadtwildnis - Korespte, Projekte und Perspoktiven MM. Diemer et a . ? 
(c) 20I0 Authors; licensee IÖW and oekom verlag. This is an article distributed under the terms of the Creative Commons Attribution Non-Commercial No Derivates License (http://creativecommons.org/licenses/by-nc-nd/3.o/), which permits unrestricted use, distribution, and reproduction in any medium, provided the original work is properly cited. 